\title{
Integração de dados radiométricos e potenciais aplicados ao estudo do embasamento adjacente à Bacia Sergipe Alagoas.
}

\author{
Sampaio, M. R. C. DGF-UFBA; Dutra, A.C. DFTMA-UFBA; Costa, A. B. DFTMA-UFBA;
}

Copyright 2019, SBGf - Sociedade Brasileira de Geofísica

This paper was prepared for presentation during the $16^{\text {th }}$ International Congress of the Brazilian Geophysical Society held in Rio de Janeiro, Brazil, 19-22 August 2019.

Contents of this paper were reviewed by the Technical Committee of the $16^{\text {th }}$ International Congress of the Brazilian Geophysical Society and do not necessarily represent any position of the SBGf, its officers or members. Electronic reproduction or storage of any part of this paper for commercial purposes without the written consent of the Brazilian Geophysical Society is prohibited.

\section{Abstract}

The objective of this study is to correlate radiometric and potential geophysical data (magnetic and gravimetric), applied to the study of the basement adjacent to the Sergipe Alagoas basin. The gravity and magnetic measurements are important to produce a geological interpreting and understanding the tectonic environment. The terrain continental boundaries are commonly recognized by contrast in all contact and knowing the gravimetric and magnetic anomaly and gradients that allows to interpret the crustal domains and their tectonic relationships with the structures in shallow sections which reside on the basement, the magnetic accommodation and lineaments are often controlled by the reactivation of basement faults. In this research, besides the primary data, the maps were constructed to the ternary map and the amplitude map of the analytic signal to aid in the interpretations. Finally, good correlation results and interesting geophysical areas were found.

\section{Introdução}

As interpretações dos métodos geofísicos de espectrometria gama, magnetometria e gravimetria têm sido utilizada como ferramenta importante nos mapeamentos geológicos, para descobrir profundidades e extensão de zonas de cisalhamento e prospecção mineral.

Com dados geofísicos potenciais obtidos a partir de medidas aeromagnéticas e de gravimetria terrestre no local pesquisado, é possível determinar além da profundidade de corpos e do embasamento, delimitar, detectar e localizar extremos de estruturas e lineamentos tectônicos a partir do tratamento destes dados. Também é possível obter contrastes de variação lateral e vertical de densidade dos domínios estudados (referências). Com os dados aerogamaespectométricos e de densidade da região, foi calculada a taxa de produção de calor radiogênico na área. Baseado nos teores de urânio, tório e potássio além de mapas derivados de grande importância interpretativa como o mapa ternário. O conhecimento das propriedades térmicas e radiométricas desempenha um papel importante na avaliação de geração de hidrocarbonetos e da história evolutiva da bacia, além da identificação de possíveis processos hidrotermais, enriquecimentos de minerais radioativos e idade relativa das formações nos domínios estudados.

O objetivo deste trabalho é correlacionar dados radiométricos e geofísicos potenciais (magnéticos e gravimétricos) aplicados ao estudo do embasamento adjacente à bacia de Sergipe Alagoas, buscando compreender o ambiente tectônico em que se situa, com intuito de refinar modelos sistemas petrolíferos para este tipo de bacia.

\section{Área de estudo}

A área de estudo encontra-se localizada no embasamento adjacente a oeste da Bacia Sergipe Alagoas, abrangendo os estados de Sergipe, Alagoas e uma parte de Pernambuco (figura 1).

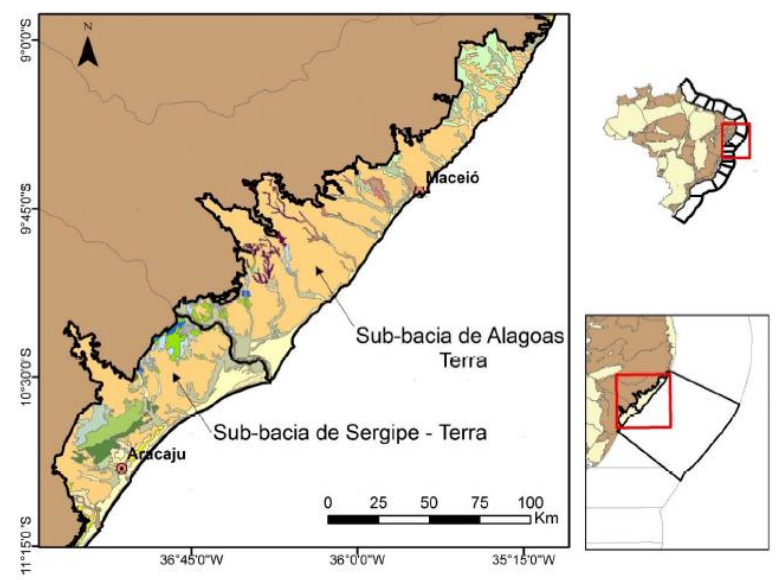

Figura 1: Localização da Bacia Sergipe Alagoas. Fonte: CPRM, folhas SC24 (Koisin et al, 2004).

Tal bacia está em avançado estado exploratório e apresenta áreas de interesse offshore e onshore.Este embasamento é resultado de uma colisão entre o Maciço Pernambuco-Alagoas e o Cráton do São Francisco, tendo influência de ambas estruturas e sua evolução tecno estrutural na interpretação dos dados obtidos. Compreende os domínios geotectônicos SalvadorItabuna-Curaçá da sub-província Cráton do São Francisco, e Estância, Canudos-Vaza-Barris, Macururé, Rio Coruripe e Pernambuco-Alagoas da sub-província Meridional da província Borborema, os quais devem constituir o embasamento da Bacia Sergipe-Alagoas (Brito Neves et al., 2000, Oliveira etal., 2010; Santos et al., 2000). 


\section{Metodologia}

Para o estudo foram utilizados dados gravimétricos oriundos de campanhas terrestres do projeto Geoterm (IF-UFBA), da Rede Nacional de Gravimetria através do banco de dados da ANP (Petrobras e outras empresas petrolíferas), da CPRM, do Instituto Brasileiro de Geografia e Estatística (IBGE) e de grupos de pesquisa. Os dados aéreos, magnéticos e radiométricos, foram cedidos pela CPRM (Companhia de Pesquisa de Recursos Minerais). Os dados de densidade utilizados foram adquiridos a partir de amostras de campanhas terrestres na região de estudo.

Após as correções e processamentos dos dados geofísicos, mapa gravimétrico da anomalia Bouguer e o mapa magnético, ambos de uma área extensa da província de Borborema que abrange toda a região do embasamento que será estudada. Foi feito ainda o mapa derivado do dado magnético, conhecido como Amplitude do Sinal Analítico (ASA) (Nabighian e Corbett, 1988).

Com os dados de espectrometria gama foram feitos mapas de teor de Potássio (K), Urânio (U) e Tório (Th) e o mapa de fator F. Para o mapa de calor radiogênico da área foi necessário a utilização dos dados terrestres de densidade. Assim foi feita uma nova interpolação com os teores de $\mathrm{U}$, Th e K, utilizando o mesmo espaçamento dos dados densidade, para que fossem compatibilizados. Foi aplicado o mesmo polígono de corte nos grids, para que tivessem o mesmo formato não ocorrendo vazios no mapa resultante. Com os valores de densidade $(\rho)$ em $\mathrm{kg} \cdot \mathrm{m}^{-3}$, de $\mathrm{K}$ em \% e os de Th e U em ppm, obteve-se o mapa de calor radiogênico $A(\mu \mathrm{W} . \mathrm{m}-3)$ através da equação:

$$
A\left(\mu W m^{-3}\right)=10^{-5} \rho\left(3,48 C_{K}+9,52 C_{U}+2,56 C_{T h}\right)
$$

Em seguida foram realizadas superposições de mapas para realizar as correlações entre os diferentes dados assim como a observação isolada de cada um deles. Estes mapas foram obtidos através do Software Oasis Montaj.

\section{Resultados}

Os principais resultados obtidos foram os mapas geofísicos citados na metodologia, a partir dos quais tiramos diversas informações de cada área e suas correlações, além da profundidade de alguns corpos de interesse e contrastes laterais de densidade. Encontramse também a delimitação de feições e lineamentos estruturais do embasamento e uma boa correlação entre a geologia estudada e os dados interpretados.

A partir de estudos do mapa gravimétrico de anomalia Bouguer (figura 2), realizando uma separação regional residual através do método algoritmo polinomial robusto de terceiro grau, foram localizadas anomalias que denotavam grandes contrastes de densidade. Os destaques são dados para altos gravimétricos na região sul-sudoeste da Província de Borborema Meridional no embasamento adjacente a sub-bacia Sergipe, no domínio Estância e próximo aos entornos do município de Boquim, onde são encontrados também granulitos do
Cráton do são Francisco aflorantes, prováveis responsáveis pela anomalia positiva, encontramos altos também no sudoeste do Domo de Itabaiana e no Domo de Simão Dias, ambos localizados no domínio CanudosVaza Barris. Há um destaque gravimétrico na região do domínio Macururé-Rio Coruripe nos entornos do município de Arapiraca, se estendendo ao domo Girau do Ponciano, com a presença de litologias gnáissicas, graníticas e migmatiticas. Os baixos ocorrem nas regiões de sedimentos fanerozoicos das bacias sedimentares e os valores chegam a um máximo de $22.5 \mathrm{mgal}$ de anomalia Bouguer.

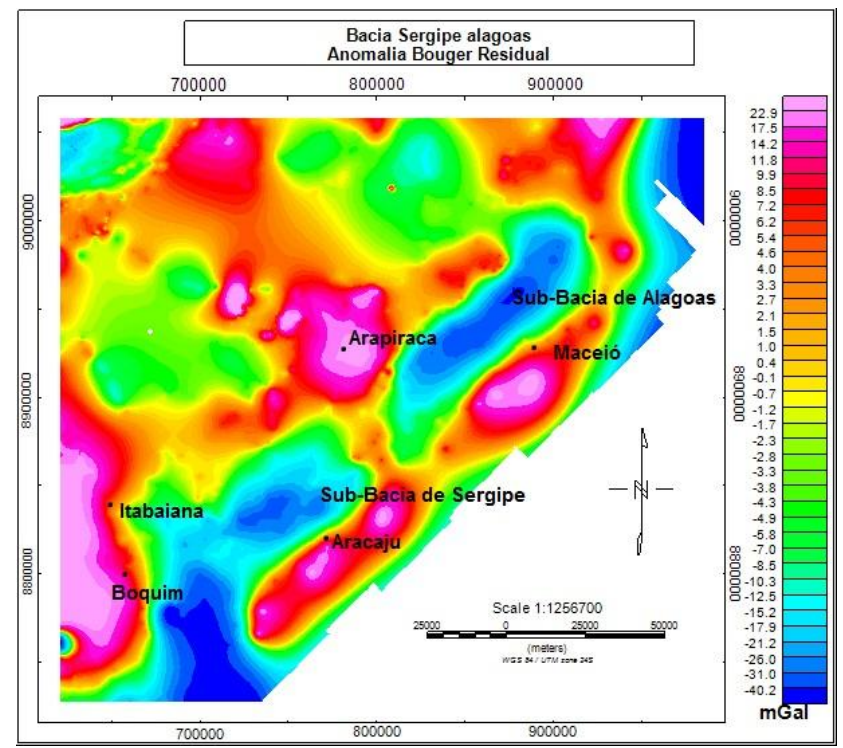

Figura 2: Mapa da anomalia bouguer residual

No dado magnético a interpretação foi estendida ao dado do ASA por sua facilidade em delinear lineamentos e delimitar estruturas anômalas ao embasamento. Por ser uma região onde houve muitos processos estruturais e tectônicos, esta região apresenta zonas de cisalhamento importantes entre elas a zona de cisalhamento Barão de Jeremoabo (ZCBJ), zona de cisalhamento Macururé (ZCM), a zona de cisalhamento São Miguel de Aleixo (ZCSMA) e a zona de cisalhamento de Itaporanga (ZCl), todas observadas no mapa do Sinal Analítico assim como - lineamento PEAL. Os principais baixos magnéticos estão nas regiões das bacias enquanto os altos estão destacados nos domos de Itabaiana e Simão Dias (delimitando seus contornos), na região do Cráton do São Francisco, ao sul de Boquim e pela região do Domínio Rio Coruripe, além de algumas regiões ao norte do mapa (figura 3). 


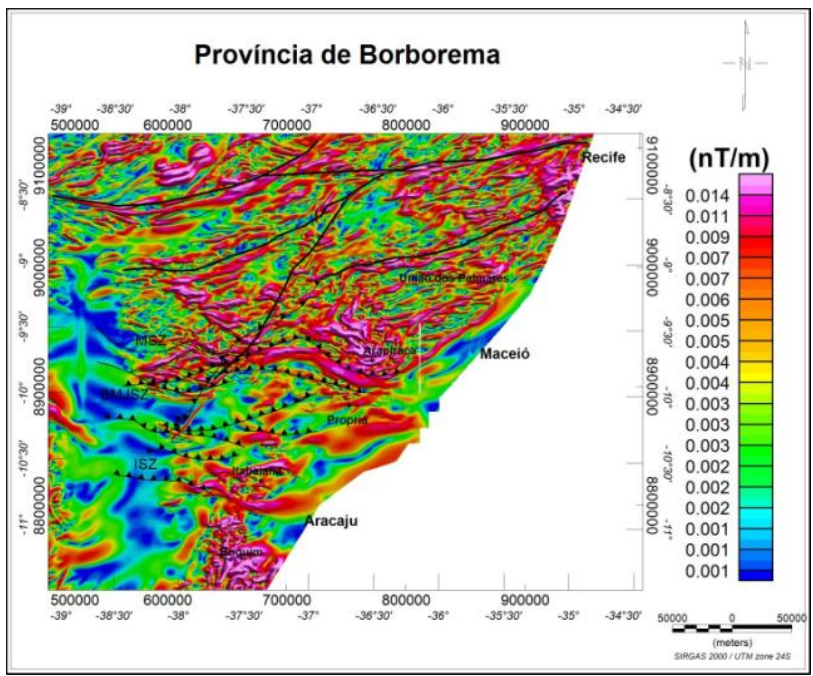

Figura 3: Mapa da Amplitude do Sinal Analítico da Província de Borborema com identificações de Zonas de Cisalhamento.

Iniciando a análise gamaespectometrica pelo mapa de teor de potássio (figura 4), identificamos altos valores concentrados na parte sudoeste da área, no domínio Estância (alto gravimétrico e magnético), na ZCBJ (identificada no mapa magnético), e de forma geral, na margem oeste do mapa ocorrendo um decrescimento à medida que vai se aproximando da área da bacia sedimentar Sergipe-Alagoas, ainda podemos observar altos teores na parte norte do Domínio Macururé, onde podemos correlacionar esses valores à presença de xistos, corpos graníticos, granodioritos e a presença de biotitas. Apresentam-se valores baixos no Domo Girau do Ponciano embora ortognaisses/granitoides como ele teoricamente apresentem valores médios a altos de potássio.

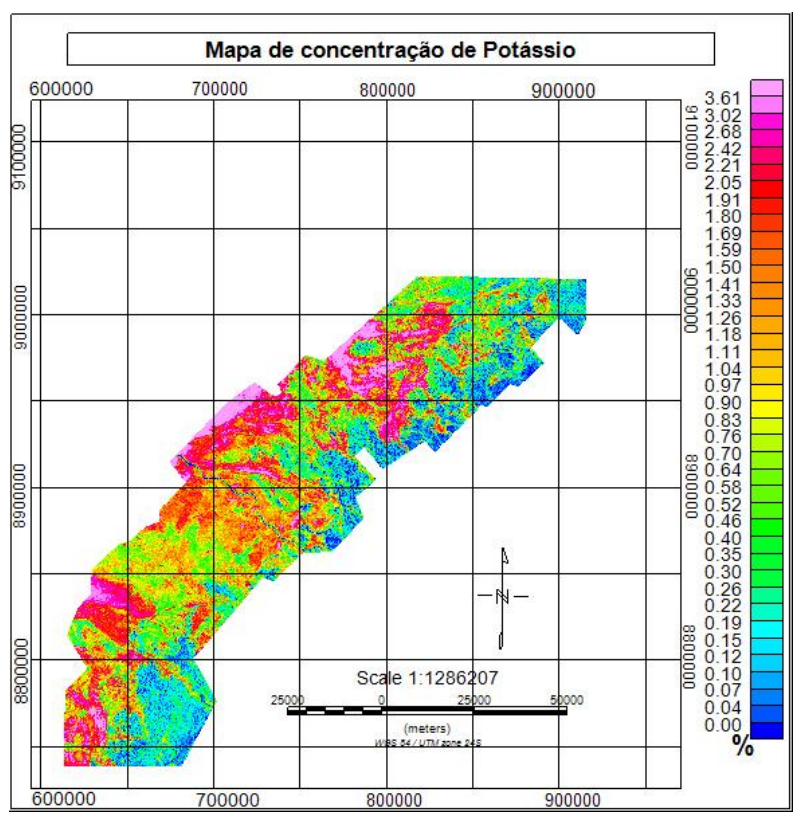

Figura 4: Mapa de teor de Potássio.
Com relação ao mapa de urânio (figura 5) temos altos valores no Norte, região do domínio Pernambuco alagoas, na ZCBJ e no domínio Rio Coruripe, com a presença de granitoides, onde ocorreram anomalias positivas nos dados gravimétricos e magnéticos estudados, enquanto nos domos de Itabaiana, Simão Dias e na região de Girau do Ponciano encontram-se baixos valores, chegando a delimitar contornos.

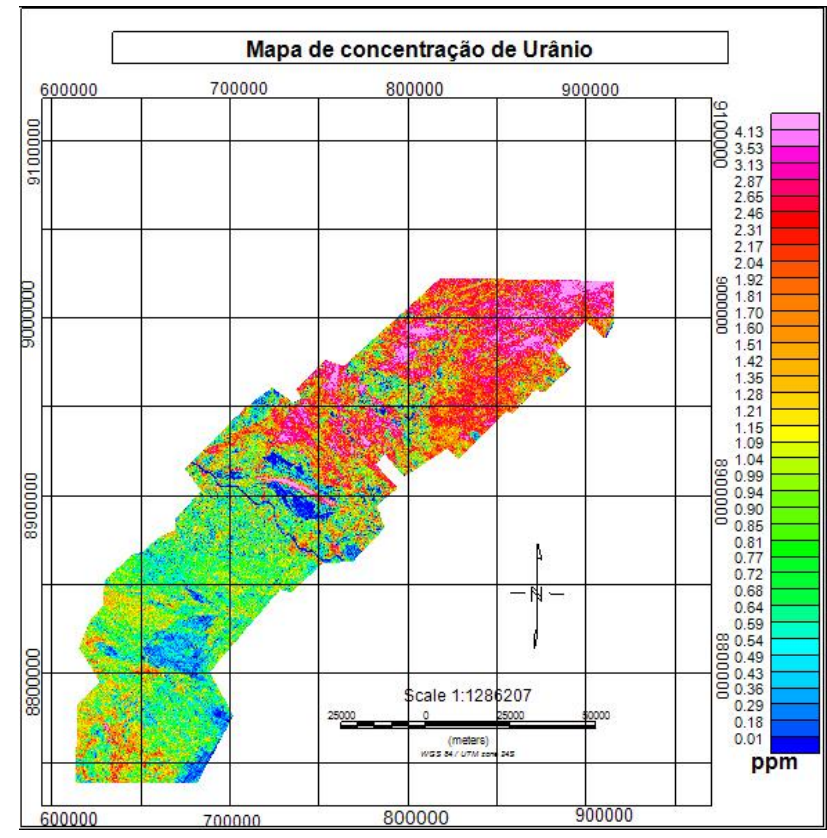

Figura 5:Mapa de teor de Urânio.

A concentração espacial de Tório e sua ocorrência é bem parecida com a do urânio como pode ser observado na figura 6 . O teor de Tório aumenta muito com o teor de sílica nas rochas e apresenta um pouco mais de concentração no domínio Canudos-Vaza Barris se comparado ao mapa de teor de Urânio (figura 5).

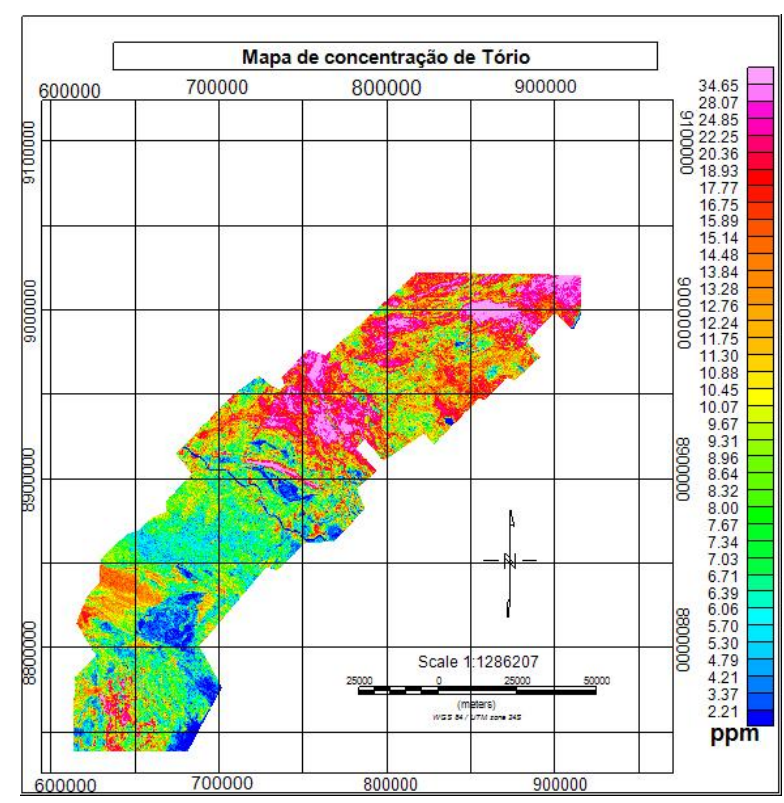

Figura 6: Mapa de teor de Tório. 
Importante destacar que a ZCBJ, facilmente delineada pelo mapa do ASA (figura 3) é destacada em todos os mapas dos radio-elementos.

Os mapas de razões não apresentaram grandes resultados interpretativos. O tório e o urânio com relação ao potássio $(\mathrm{Th} / \mathrm{K}$ e $\mathrm{U} / \mathrm{K})$ apresentam altos na margem leste da bacia enquanto o Tório e o Urânio têm uma relação $(\mathrm{U} / \mathrm{Th})$ pouco variada entre eles apresentando baixos pelo Jirau do Ponciano e altos ao norte do mapa.

O mapa de fator $F$ (figura 7), utilizado na identificação de hidrotermalismo identificou um alto na área da ZCBJ, logo, ao que tudo indica, pode realmente ter ocorrido um processo hidrotermal na área e ate mesmo gerado uma zona mineralizante que deveria ser pesquisada mais a fundo.

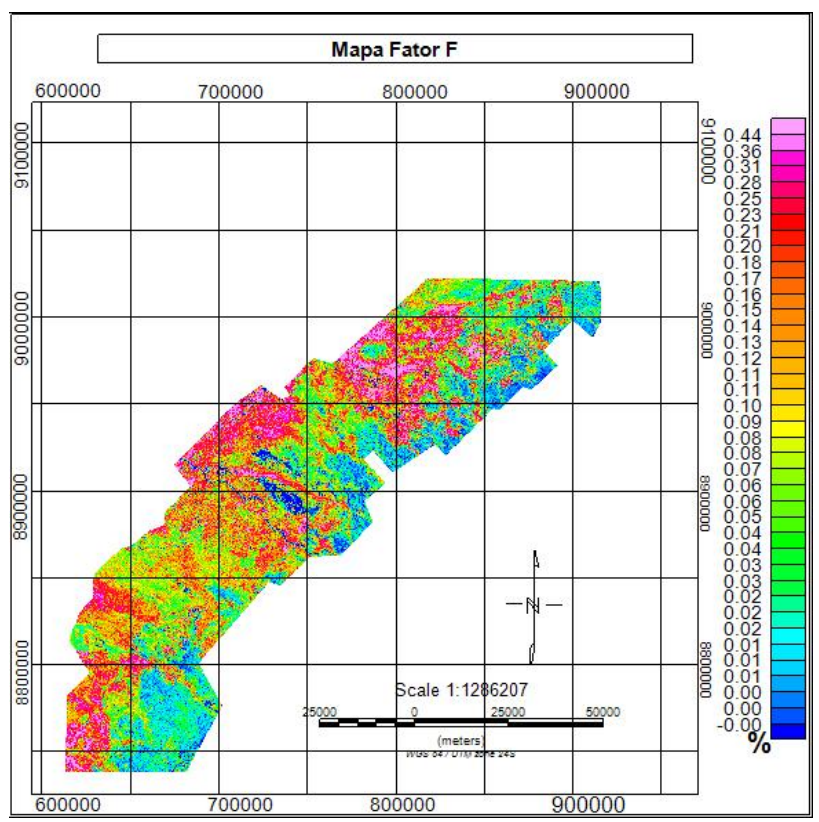

Figura 7: Mapa fator F da área estudada.

O mapa ternário foi gerado utilizando o padrão RGB, que associa as cores vermelha ( $R$ ), verde $(G)$ e azul (B) ao $\mathrm{K}$, Th e $\mathrm{U}$ respectivamente. Os resultados obtidos a seguir podem ser observados na figura 8 . Nas zonas onde prevalece a cor branca, ou seja, em alguns pontos ao norte, pelo domínio Rio Coruripe e, ao longo da ZCBJ temos altas concentrações de todos os radioelementos estudados (K, U, Th), comumente associada a processos de hidrotermalismo. Nas zonas mais escuras localizadas mais para o Centro-Sul, têm-se menores concentrações dos elementos, se a cor tende ao vermelho encontra-se mais potássio, se tender para o verde o elemento Tório é o que se encontra mais abundante e caso a cor tenda para o azul, teremos maiores concentrações de Urânio.

Nos locais circulados de amarelo temos os domos mais estudados desta região, mais ao sudoeste encontra-se o Domo de Simão Dias (3) e próximo a este temos o Domo de Itabaiana (2), ambos com maiores contagens de potássio ainda que com baixos valores de todos os elementos. Mais ao Norte em contato com a ZCBJ temos o Domo de Girau do Ponciano (1) com as mesmas condições gamaespectométricas dos outros domos.
Podemos perceber que as estruturas são muito bem delineadas superficialmente no mapa Ternário.

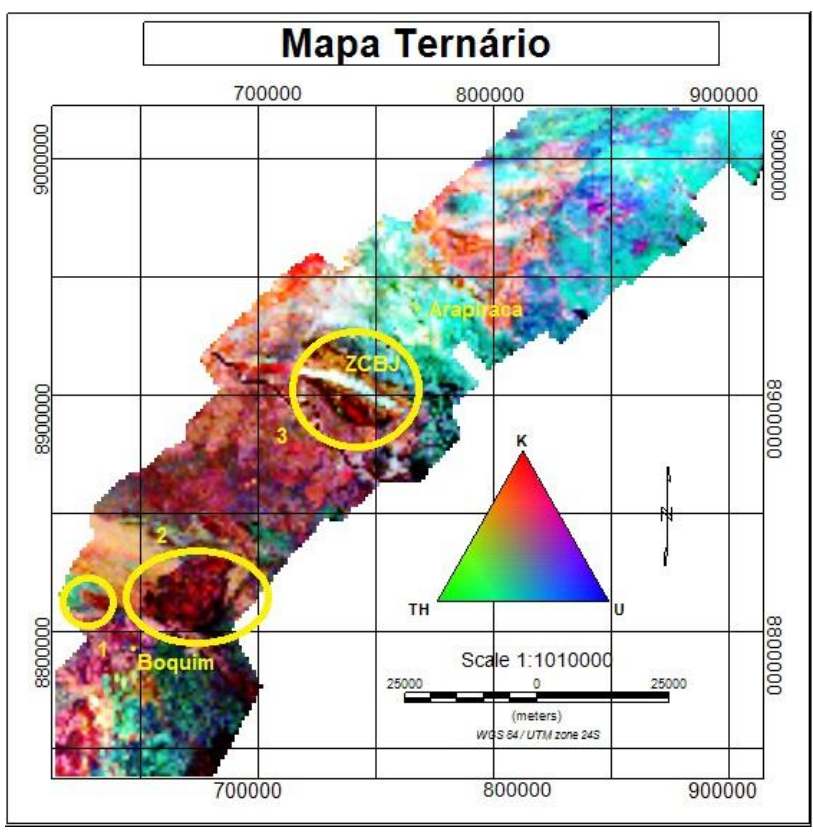

Figura 8: Mapa Ternário RGB da área estudada.

Por fim, o mapa do calor radiogênico (figura 9) produzido nos deu resultados com taxas maiores de produção de calor concentradas ao norte da área de estudo, onde afloram corpos graníticos, assim como ocorre altos no domínio Rio Coruripe e na ZCBJ, enquanto na região do domínio Macururé estão os valores mais baixos e ficam menores ainda nas estruturas dômicas de Itabaiana (2), Simão Dias (3) e Girau do Ponciano (1), logo podemos chegar a conclusões de que estas estruturas mais "frias" são mais antigas que o embasamento adjacente a bacia, denotando processos tectônicos que resultaram em soerguimentos. A produção de calor é baixa nos domínios Estância e Canudos-Vaza-Barris e os valores variam de 0.24 a $3.32 \mu \mathrm{W} / \mathrm{m}^{3}$ em toda a área. Tais dados de produção radiogênica serão reaproveitados em um próximo estudo para que se possa mapear o fluxo de calor do embasamento e consequente avaliação de potencial gerador de hidrocarbonetos na área estudada. 


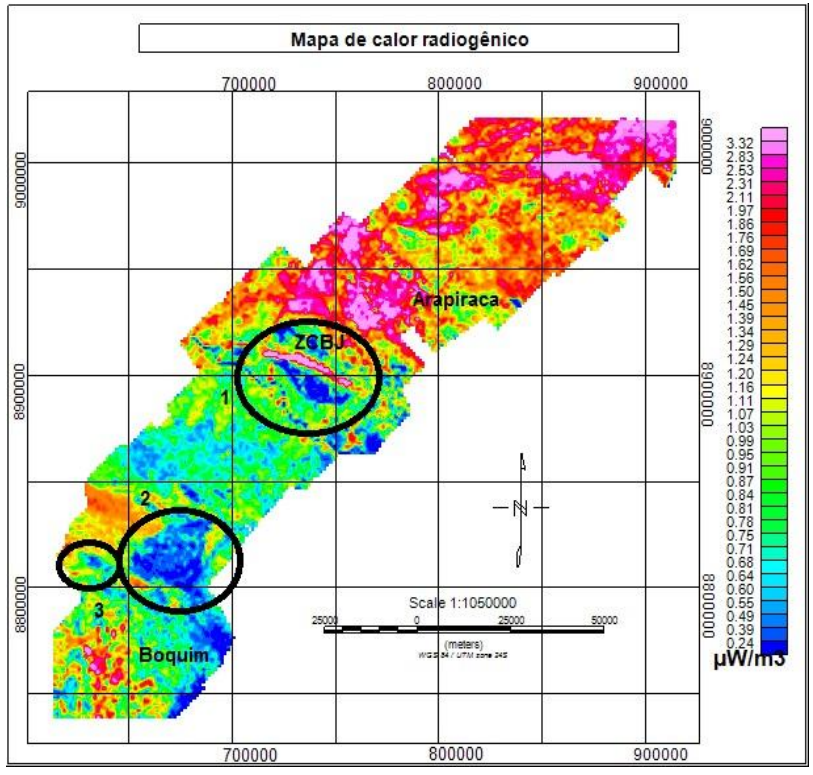

Figura 9: Mapa de calor radiogênico da área estudada.

\section{Conclusões}

Apesar da diferença de cobertura espacial entre os mapas geofísicos potenciais e os de espectometria gama, não houve problemas quanto às correlações visto que, a menor área utilizada era suficiente para estudar os mesmos corpos de interesse dos dados de maior cobertura.

Correlacionando o mapa da Amplitude do Sinal Analítico e os radiométricos foi possível demarcar lineamentos e estruturas no mapa magnético, dentre as quais, a ZCBJ foi a que mais se destacou, pois ela concordou com uma estrutura bastante acentuada por apresentar altos teores de todos os radioelementos estudados e alta taxa de produção de calor radiogênico. Além disso, como mostrado pelo mapa ternário, locais que apresentam a cor branca tem maiores chances de ter ocorrido processos hidrotermais, e percebendo que existe anomalias magnéticas no mesmo local e anomalias gravimétricas indicando alta densidade e possíveis minerais magnéticos, esta zona deveria ser explorada em busca de minérios.

A correlação dos mapas também forneceu excelentes resultados quanto as delimitações de estruturas como os domos de Itabaiana e o de Simão Dias, tanto no mapa de calor radiogenico e ternário quanto no mapa magnético. Os dados gravimétricos foram de extrema importância na identificação de anomalias de densidade, mapeando suas variações laterais de densidade nos diferentes domínios. Finalmente o estudo foi satisfatório e os dados de teores de elementos radiomètricos e os mapas de dados geofísicos potenciais foram bem utilizados na avaliação do embasamento adjacente à Bacia SergipeAlagoas, inclusive denotando interesses econômicos fora da própria bacia, a qual era o objeto de estudo inicial.

\section{Agradecimentos}

Agradecemos ao Promob - Cenpes - Petrobras pelo apoio através do projeto Petrobras 0050.0082543.13-9 e á CPRM, ANP e IBGE pelos dados fornecidos e a todos os pesquisadores contribuintes deste projeto.

\section{Referências}

ARGOLLO, R. M. ; Marinho, M. M. ; COSTA, ALEXANDRE BARRETO ; SAMPAIO FILHO, H. A. ; SANTOS, E. J. ; COUTINHO, L. F. C. . Modelo crustal e fluxo de calor nos domínios Estância, Canudos-VazaBarris e Macururé adjacentes às bordas emersas da Bacia Sergipe-Alagoas. Boletim de Geociências da Petrobras (Online), v.20, p. 283, 2012.

BOEKER, Liége Almeida et al. Taxa de produçăo de calor radiogęnico e propriedades térmicas, nas diferentes litologias do Embasamento Adjacente à Bacia de Sergipe. Domínio Canudos-Vaza-Barris e Domos de Itabaiana-Simăo Dias. In: V Simpósio Brasileiro de Geofísica. 2012.

CUNHA, L. O. ; DUTRA, A. C. ; COSTA, A. B. . Estudo de zonas com alteração hidrotermal através dos métodos aerogeofísicos no leste da Província Borborema. In:47ํㅜ Congresso Brasileiro de Geologia, 2014, Salvador. Anais do 47ํㅡㄹ Congresso Brasileiro de Geologia, 2014.

DUTRA, Alanna Costa. Determinação da espessura da interface sedimento-embasamento da Bacia SergipeAlagoas a partir da assinatura gravimétrica. Revista de Geociências do Nordeste, v. 3, n. 1, p. 28-47, 2017.

FERREIRA, Francisco José Fonseca. Gamaespectrometria Aérea e sua Aplicação na Cartografia Geológica. Tese de Doutorado. Universidade Federal do Paraná.

MENDES, V. A.; BRITO, M. F. L.; PAIVA, I. P. Programa Geologia do Brasil-PGB. Arapiraca. Folha SC. 24-XD. Estados de Sergipe, Alagoas e Pernambuco. CPRMServiço Geológico do Brasil, Recife. Escala, 2009.

RIBEIRO, Vanessa Biondo; MANTOVANI, Marta SM; LOURO, Vinicius Hector Abud. Aerogamaespectrometria e suas aplicações no mapeamento geológico. Terræ Didatica, v. 10, n. 1, p. 29-51, 2014. 